\title{
ON A CONJECTURE OF VUKMAN
}

\author{
QING DENG \\ Department of Mathematics \\ Southwest China Normal University \\ Chongqing 630715, P.R CHINA
}

(Received October 27, 1993 and in revised form October 30,1995)

\begin{abstract}
Let $R$ be a ring A bi-additive symmetric mapping $d: R \times R \rightarrow R$ is called a symmetric bi-derivation if, for any fixed $y \in R$, the mapping $x \rightarrow D(x, y)$ is a derivation The purpose of this paper is to prove the following conjecture of Vukman

Let $R$ be a noncommutative prime ring with suitable characteristic restrictions, and let $D: R \times R \rightarrow R$ and $f: x \rightarrow D(x, x)$ be a symmetric bi-derivation and its trace, respectively Suppose that $f_{n}(x) \in Z(R)$ for all $x \in R$, where $f_{k+1}(x)=\left[f_{k}(x), x\right]$ for $k \geq 1$ and $f_{1}(x)=f(x)$, then $D=0$
\end{abstract}

KEY WORDS AND PHRASES: Prime ring, centralizing mapping, symmetric bi-derivation. 1991 AMS SUBJECT CLASSIFICATION CODES: Primary 16W25; Secondary 16N60

\section{INTRODUCTION}

Throughout this paper, $R$ will denote an associative ring with center $Z(R)$. We write $[x, y]$ for $x y-y x$, and $I_{a}$ for the inner derivation deduced by a A mapping $D: R \times R \rightarrow R$ will be called symmetric if $D(x, y)$ holds for all pairs $x, y \in R \quad$ A symmetric mapping is called a symmetric biderivation, if $D(x+y, z)=D(x, z)+D(y, z)$ and $D(x y, z)=D(x, z) y+x D(y, z)$ are fulfilled for all $x, y \in R$ The mapping $f: R \rightarrow R$ defined by $f(x)=D(x, x)$ is called the trace of the symmetric bi-derivation $D$, and obviously, $f(x+y)=f(x)+f(y)+2 D(x, y)$ The concept of a symmetric biderivation was introduced by Gy Maksa in [1,2] Some recent results concerning symmetric bi-derivations of prime rings can be found in Vukman [3,4]. In [4], Vukman proved that there are no nonzero symmetric bi-derivations $D$ in a noncommutative prime ring $R$ of characteristic not two and three, such that $[[D(x, x), x], x] \in Z(R)$. The following conjecture was raised Let $R$ be a noncommutative prime ring of characteristic different from two and three, and let $D: R \times R \rightarrow R$ be a symmetric bi-derivation. Suppose that for some integer $n \geq 1$, we have $f_{n}(x) \in Z(R)$ for all $x \in R$, where $f_{k+1}(x)=\left[f_{k}(x), x\right]$ for $k=1,2, \ldots$, and $f_{1}(x)=D(x, x)$ Then $D=0$.

The purpose of this paper is to prove this conjecture under suitable characteristic restrictions

\section{THE RESULTS}

THEOREM 1. Let $R$ be a prime ring of characteristic different from two Suppose that $R$ admits a nonzero symmetric bi-derivation. Then $R$ contains no zero divisors.

PROOF. It is sufficient to show that, $a^{2}=0$ for $a \in R$ implies $a=0$ We need three steps to establish this

LEMMA A. If $D(a, *) \neq 0$, then $D(a, *)=\mu I_{a}$, where $\mu \in C$, the extended centroid of $R$

PROOF. Since $D\left(a^{2}, x\right)=D(0, x)=0$, we have 


$$
a D(a, x)+D(a, x) a=0 \text { for all } x \in R .
$$

Replacing $x$ by $x y$, we obtain

$$
I_{a}(x) D(a, y)=D(a, x) I_{a}(y) \quad \text { for all } \quad x \in R
$$

and replacing $y$ by $y z$, we get

$$
I_{a}(x) y D(a, z)=D(a, x) y I_{a}(z), x, y, z \in R .
$$

Since $D(a, *) \neq 0$, we may suppose that $D(a, z) \neq 0$ for a fixed $z \in R$. Obviously $I_{a}(Z) \neq 0$ By (2 1), and by [5, Lemma 1.3.2], there exist $\mu(x)$ and $\nu(x)$ in $C$, either $\mu(x)$ or $\nu(x)$ being not zero, such that $\mu(x) I_{a}(x)+\nu(x) D(a, x)=0$. If $\nu(x) \neq 0$ then $D(a, x)=\frac{-\mu(x)}{\nu(x)} I_{a}(x)$; on the other hand, if $\nu(x)=0$ then $\mu(x) I_{a}(x)=0$ and $I_{a}(x)=0$, using (2.1) and $I_{a}(z) \neq 0$, so $D(a, x)=0$. In any event, we have $D(a, x)=\mu(x) I_{a}(x) \quad$ Hence (2.1) implies $(\mu(x)-\mu(z)) I_{a}(x) y I_{a}(z)=0 \quad$ It follows that either $I_{a}(x)=0$ or $\mu(x)=\mu(z) \quad$ By $(2.1)$, the former implies $D(a, x)=0$ and $D(a, x)=\mu(z) I_{a}(x)$ In both cases, we get $D(a, x)=\mu(z) I_{a}(x)$ for all $x \in R$, and $0 \neq \mu(z)$ being fixed

The fixed element $\mu$ in Lemma $\mathrm{A}$ is somewhat dependent on $a$, we write it as $\mu_{a}$ For any given $r \in R$ ara satisfies our original hypotheses on $a$; therefore for each $r \in R$, either $D($ ara,*) $=0$ or $d($ ara,$*)=\mu_{\text {ara }} I_{\text {ara }}$, where $\mu_{\text {ara }} \neq 0$

LEMMA B. If $D($ ara,$*) \neq 0$, then $\mu_{\text {ara }}=\mu_{a}$.

PROOF. $D($ ara $*) \neq 0$ implies ara $\neq 0$ Suppose that $D(a, *)=0$, then $D(\operatorname{ara}, x)=D(a, x)$ ra+ $a D(r, x) a+\operatorname{ar} D(a, x)=a D(r, x) a$; but $D($ ara,$x)=\mu_{\text {ara }} I_{\text {ara }}(x)=\mu_{\text {ara }}($ arax $-x a r a)$, so that $\mu_{\text {ara }}(\operatorname{arax}-x a r a)=a D(r, x) a$ Right-multiplying the last equation by $a$, we have $\mu_{\text {ara } a} a r a x a=0$ for all $x \in R$. It follows that $a r a=0$, a contradiction Therefore $D(a, *)=\mu_{a} I_{a}$, and consequently,

$$
D(\operatorname{ara}, x)=\mu_{a} I_{a}(x) r a+a D(r, x) a+a r \mu_{a}(x) ;
$$

and right-multiplying this equation by $a$ yields

$$
D(a r a, x) a=\mu_{a} \text { araxa for all } x \in R .
$$

Hence $\mu_{\text {ara }}$ araxa $=\mu_{a}$ araxa, immediately $\mu_{\text {ara }}=\mu_{a}$.

LEMMA C. If $a^{2}=0$, then $a=0$.

PROOF. Let $S=\left\{r \in R \mid D(\right.$ ara,$\left.*)=\mu_{\text {ara }} I_{\text {ara }}, \mu_{\text {ara }} \neq 0\right\}$ and $T=\{r \in R \backslash D($ ara,$*)=0\}$ By Lemma $\mathrm{A}$ and $\mathrm{B}, R=S \cup T$ and $S$ and $T$ are additive subgroups of $R$ We conclude that either $S=R$ or $T=R$.

Suppose that $S=R$ Lemma A gives, either $D(a, *)=0$ or $D(a, *)=\mu_{a} I_{a}$. If $D(a, *)=0$, then $D(\operatorname{ara}, x)=a D(r, x) a$, for all $r, x \in R$, and $D($ ara, $x) a=0$. It follows that $\mu_{a}$ araxa $=0$. Since $\mu_{a}=\mu_{\text {ara }} \neq 0$, we have $a=0$ If $D(a, *)=\mu_{a} I_{a}$, then the equation

$$
D(a r a, y a)=D(a, y a) r a+a D(r, y a) a+a r D(a, y a)
$$

gives $\mu_{a}$ araya $=2 \mu_{a}$ ayara $+\mu_{a}$ araya. Hence we get ayara $=0$, and $a=0$ again

We suppose henceforth that $T=R$ If $D(a, *)=0$, then $D(a x a, y z)=a D(x a, y z)=0$, and ay $D(x a, z)=0$. Thus $D(x a, z)=D(x, z) a=0$, and $D(x, y) z a=D(x, y z) a=0$ Since $D \neq 0$, we then get $a=0$. If $D(a, *)=\mu_{a} I_{a}$, then, right-multiplying the equation $D(a x a, y)=0$ by $a$, we obtain $\mu_{a} a x a y a=\operatorname{ax} D(a, y) a=0$, and $a=0$ again. The proof of the theorem is complete

In order to prove Vukman's conjecture, we need the following proposition.

PROPOSITION. Let $n$ be a positive integer; let $R$ be a prime ring with char $R=0$ or char $R>n$; and let $g$ be a derivation of $R$ and $f$ the trace of a symmetric bi-derivation $D \quad$ For $i=1,2, \ldots, n$, let $F_{2}(X, Y, Z)$ be a generalized polynomial such that, $F_{2}(k x, f(k x), g(k x))=k^{2} F_{2}(x, f(x), g(x))$ for all $x \in R$ for $k=1,2, \ldots, n$. Let $a \in R$, and (a) the additive subgroup generated by $a$ If for all $x \in(a)$, 


$$
F_{a}(x, f(x), g(x))+F_{n-1}(x, f(x), g(x))+\ldots+F(x, f(x), g(x)) \in Z(R),
$$

then $F_{\imath}(a, f(a), g(a)) \in Z(R)$ for $i=1,2, \ldots, n$

This proposition can be proved by replacing $x$ by $a, 2 a, \ldots, n a$ in (2.2) and applying a standard "Van der Monde argument "

THEOREM 2. Let $n$ be a fixed positive integer and $R$ be a prime ring with char $R=0$ or char $R>n+2$ Let $f_{k+1}(x)=\left[f_{k}(x), x\right]$ for $k>1$, and $f_{1}(x)=f(x)$ the trace of a symmetric biderivation $D$ of $R$. If $f_{n}(x) \in Z(R)$ for all $x \in R$, then either $D=0$ or $R$ is commutative

PROOF. Linearizing $f_{n}(x) \in Z(R)$, we obtain

$$
[[\ldots[f(x)+f(y)+2 D(x, y), x-y], \ldots x+y], x+y] \in Z(R) ;
$$

and using the Proposition, we get

$$
\begin{aligned}
& {[\ldots[[f(x), y], x], \ldots, x]+[\ldots[[f(x), x], y], \ldots x]+\ldots+[\ldots[f(x), x], \ldots y] } \\
&+2[\ldots[[D(x, y), x], x], \ldots, x] \in Z(R),
\end{aligned}
$$

equivalently,

$$
\begin{aligned}
& (-1)^{n-2} I_{x}^{n-2}\left(\left[f_{1}(x), y\right]\right)+(-1)^{n-3} I_{x}^{n-3}\left(\left[f_{s}(x), y\right]\right)+\ldots \\
& +\left[f_{n-1}(x), y\right]+2(-1)^{n-1} I_{x}^{n-1}(D(x, y)) \in Z(R)
\end{aligned}
$$

Noting that

$$
\begin{aligned}
(-1)^{n-2} I_{x}^{n-2}\left(\left[f_{1}(x), x^{2}\right]\right) & =(-1)^{n-3}\left(\left[f_{2}(x), x^{2}\right]\right)=\ldots \\
& =\left[f_{n-1}(x), x^{2}\right]=(-1)^{n-1} I_{x}^{n-1}\left(D\left(x, x^{2}\right)\right)=2 f_{n}(x) x,
\end{aligned}
$$

and replacing $y$ by $x^{2}$ in (2.3), we then get $2(n+1) f_{n}(x) x \in Z(R)$ Since $f_{n}(x) \in z(R)$, it follows that $f_{n}(x)=0$

The linearization of $f_{n}(x)=0$ gives

$$
\begin{aligned}
& (-1)^{n-2} I_{x}^{n-1}\left(\left[f_{1}(x), y\right]\right)+(-1)^{n-3} I_{x}^{n-3}\left(\left[f_{2}(x), y\right]\right) \\
& \quad+\ldots+\left[f_{n-1}(x), y\right]+2(-1)^{n-1} I_{x}^{n-1}(D(x, y))=0 .
\end{aligned}
$$

Since $\quad I_{x}^{n-k}\left(\left[f_{k-1}(x), x y\right]\right)=x I_{x}^{n-1}\left(\left[f_{k-1}(x), y\right]\right)+I_{k}^{n-k}\left(f_{k}(x) y\right) \quad$ for $\quad k=2,3, \ldots, n, \quad$ and $I_{x}^{n-1}(D(x, x y))=x I_{x}^{n-1}(D(x, y))+I_{x}^{n-1}\left(f_{1}(x) \cdot y\right)$. Substituting $x y$ for $y$ in (24), we have

$$
\begin{aligned}
(-1)^{n-2} I_{x}^{n-2}\left(f_{2}(x) y\right) & +(-1)^{n-3} I_{x}^{n-3}\left(f_{3}(x) y\right)+\ldots+(-1) \\
\left(I_{x}\left(f_{n-1}(x) y\right)+2(-1)^{n-1}\right) I_{x}^{n-1}\left(f_{1}(x) y\right) & =0 .
\end{aligned}
$$

Taking $y=f_{n-2}(x)$, applying $I_{x}^{k}(a b)=\sum_{j=0}^{k}\left(\begin{array}{c}k \\ j\end{array}\right) I_{x}^{k-\jmath}(a) I_{x}^{\jmath}(b)$ and noting $I_{x}^{2}\left(f_{j}(x)\right)=0$ for $i+j \geq n$, we then conclude that

$$
\begin{aligned}
2(-1)^{n-1}\left(\begin{array}{c}
n-1 \\
1
\end{array}\right) I_{x}^{n-2}\left(f_{1}(x) I_{x}\left(f_{n-2}(x)\right)\right)+(-1)^{n-2}\left(\begin{array}{c}
n-2 \\
1
\end{array}\right) & I_{x}^{n-3}\left(f_{2}(x)\right) I_{x}\left(f_{n-2}(x)\right)+\ldots \\
& +(-1) f_{n-1}(x) I_{x}\left(f_{n-2}(x)\right)=0
\end{aligned}
$$

But $(-1)^{k} I_{x}^{k-1}\left(f_{n-k}(x)\right) I_{x}\left(f_{n-2}(x)\right)=\left(f_{n-1}(x)\right)^{2}$, so $(n+2)(n-1)\left(f_{n-1}(x)\right)^{2}=0$, and by the hypotheses on the characteristic, we get $\left(f_{n-1}(x)\right)^{2}=0$ Suppose that $D \neq 0$ By Theorem 1, $f_{n-1}(x)=0$, and by induction, $f_{2}(x)=[f(x), x]=0$ Using Vukman [3, Theorem 1], $R$ is commutative, we complete the proof of Theorem 2

THEOREM 3. Let $n>1$ be an integer and $R$ be a prime ring with char $R=0$ or char $R>n+1$, and let $f(x)$ be the trace of a symmetric bi-derivation $D$ of $R$ Suppose that $\left[x^{2}, f(x)\right] \in Z(R)$ for all $x \in R$ In this case either $D=0$ or $R$ is commutative 
PROOF. Using the condition $\left[x^{n}, f(x)\right] \in Z(R)$, we get $\left[x^{2 n}, f\left(x^{2}\right)\right] \in Z(R)$, and

$$
\left[x^{2 n}, f(x)\right] x^{2}+x^{2}\left[x^{2 n}, f(x)\right]+2 x\left[x^{2 n}, f(x)\right] x \in Z(R) .
$$

Noting that $\left[x^{2 n}, f(x)\right]=2\left[x^{n}, f(x)\right] x^{n}$, we now have from (2.5) that $8\left[x^{n}, f(x)\right] x^{n+2} \in Z(R)$ Thus either $\left[x^{n}, f(x)\right]=0$ or $x^{n+2} \in Z(R)$.

But linearizing $\left[x^{n}, f(x)\right] \in Z(R)$ and applying the Proposition gives

$$
\left[x^{n-1} y+x^{n-2} y x+\ldots+y x^{n-1}, f(x)\right]+2\left[x^{n}, D(x, y)\right] \in Z(R)
$$

for all $x, y \in R$, and taking $y=x^{3}$, yields

$$
n\left[n^{n+2}, f(x)\right]+6\left[x^{n}, f(x)\right] x^{2} \in Z(R) .
$$

Suppose that $\left[x^{n}, f(x)\right] \neq 0$, then $x^{n+2} \in Z(R)$ and $\left[x^{n}, f(x)\right] x^{2} \in Z(R)$, hence $x^{2} \in Z(R)$ Now this condition, together with $x^{n+2} \in Z(R)$, implies either $x^{2}=0$ or $x^{n} \in Z(R)$, so that in each event, $\left[x^{n}, f(x)\right]=0$

Linearizing $\left[x^{n}, f(x)\right]=0$ and using the Proposition, we have

$$
\left[x^{n-1} y+x^{n-2} y x+\ldots+y x^{n-1}, f(x)\right]+2\left[x^{n}, D(x, y)\right]=0
$$

Replacing $y$ by $x^{2}$ yields $n\left[x^{n+1}, f(x)\right]=0$, hence $[x, f(x)] x^{n}=0 \quad$ If $D \neq 0$, then by Theorem 1 , $[x, f(x)]=0$, and by Vukman [3, Theorem 1], $R$ is commutative This completes the proof

ACKNOWLEDGMENT. I am indebted to Prof M. N Daif for his help I would also like to thank the referee for his valuable suggestions.

\section{REFERENCES}

[1] MAKSA, GY., A remark on symmetric biadditive functions having nonnegative diagonalization, Glas. Mat. 15 (1980), 279-282.

[2] MAKSA, GY, On the trace of symmetric bi-derivations, C. R. Math. Rep. Acad. Canada 9 (1987), 303-307

[3] VUKMAN, J., Symmetric bi-derivations on prime and semiprime rings, Aequationes Math. 38 (1989), 245-254

[4] VUKMAN, J, Two results concerning symmetric bi-derivations on prime rings, Aequationes Math. 40 (1990), 181-189.

[5] HERSTEIN, I.N., Rings with Involution, University of Chicago Press, 1976 


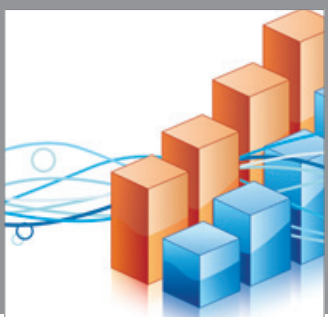

Advances in

Operations Research

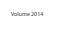

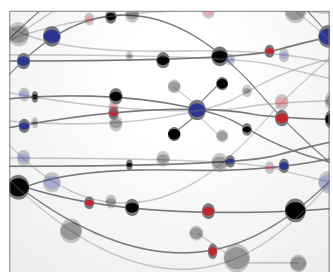

\section{The Scientific} World Journal
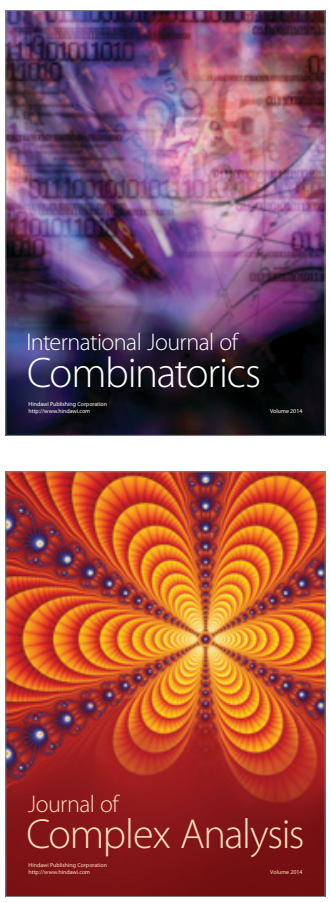

International Journal of

Mathematics and

Mathematical

Sciences
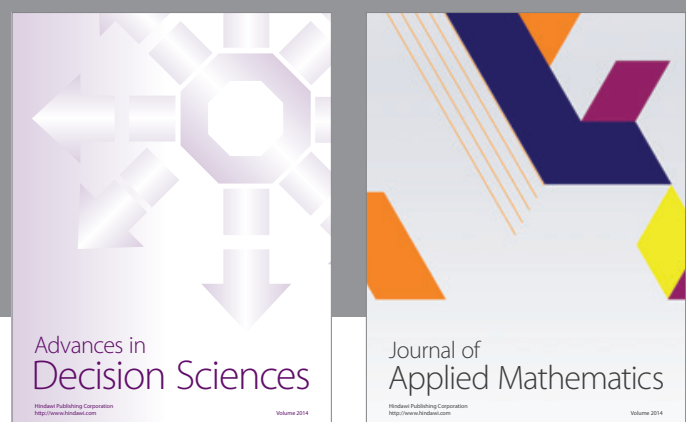

Journal of

Applied Mathematics
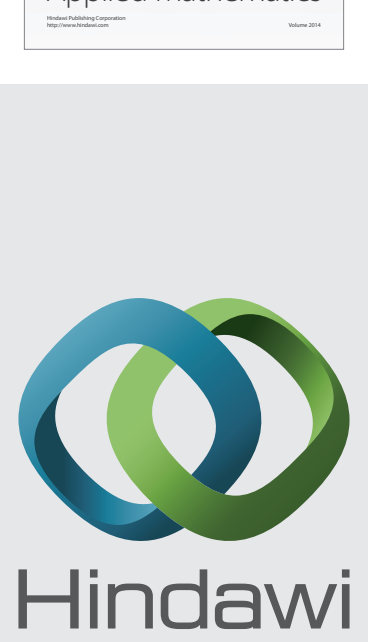

Submit your manuscripts at http://www.hindawi.com
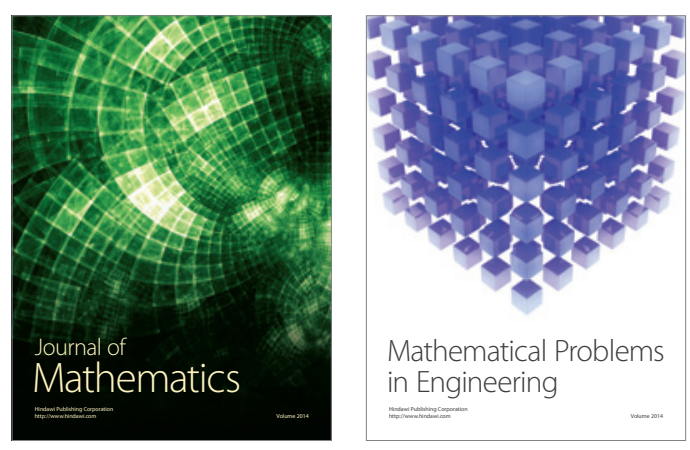

Mathematical Problems in Engineering
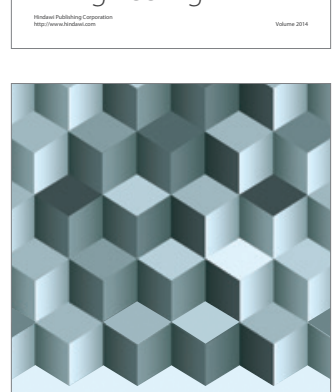

Journal of

Function Spaces
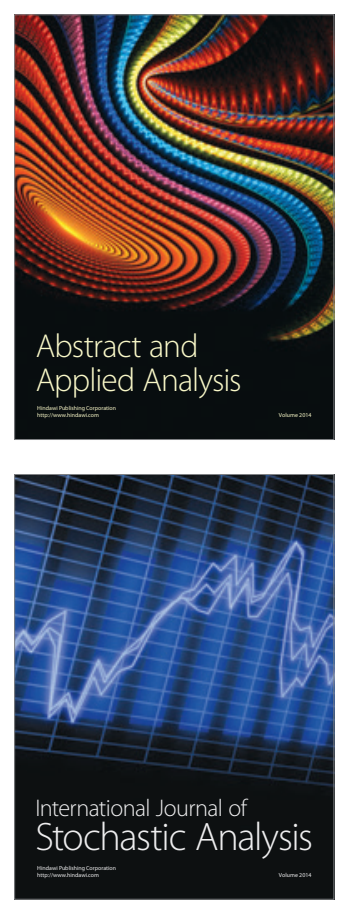

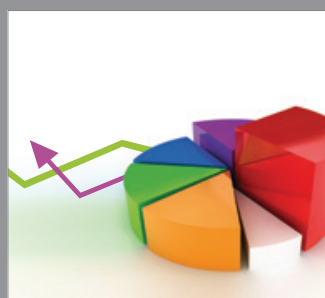

ournal of

Probability and Statistics

Promensencen
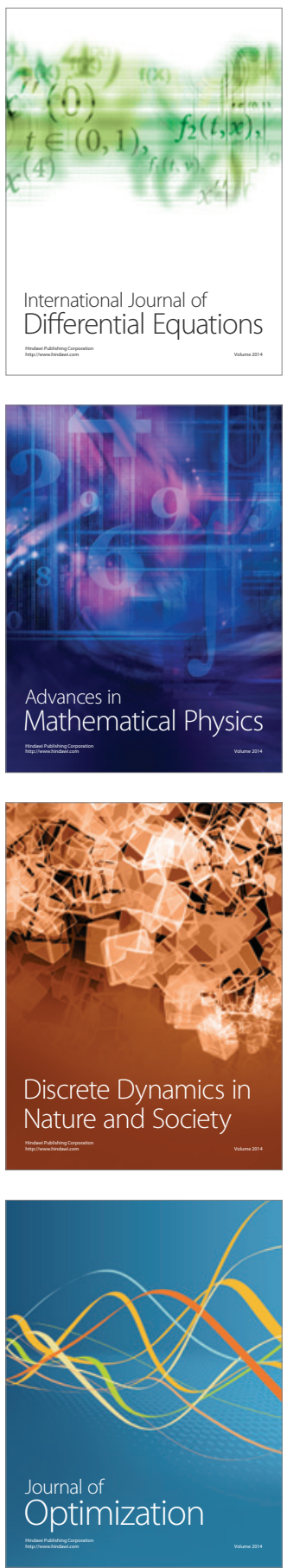